\title{
Structure and Dynamics of Spontaneous and Induced ELMs on ASDEX Upgrade
}

\author{
J. Neuhauser, V. Bobkov, G. D. Conway, R. Dux, T. Eich, M. Garcia-Munoz, A. Herrmann, \\ L. D. Horton, A. Kallenbach, S. Kalvin ${ }^{1}$, G. Kocsis ${ }^{1}$, B. Kurzan, P. T. Lang, M. Maraschek, \\ H. W. Mueller, H. D. Murmann, R. Neu, A. G. Peeters, M. Reich, V. Rohde, A. Schmid, W. \\ Suttrop, M. Tsalas ${ }^{2}$, E. Wolfrum, and the ASDEX Upgrade Team
}

\author{
Max-Planck-Institut für Plasmaphysik, EURATOM Association, Garching, Germany \\ ${ }^{1}$ KFKI-RMKI, EURATOM Association, Budapest, Hungary \\ ${ }^{2}$ NCRS Demokritos, EURATOM Association, Athens, Greece
}

e-mail: Josef.Neuhauser@ipp.mpg.de

\begin{abstract}
In order to assess the contribution of edge localized modes (ELMs) to plasma-wall interaction in future fusion experiments like ITER, a sound experimental data base for model validation and extrapolation, and, to be prepared for the unfavourable case, the development of tools for ELM mitigation are required. On ASDEX Upgrade a large amount of experimental information has been accumulated from various diagnostics on the structure and dynamics of natural as well as pellet induced ELMs, and on related wall effects. In this paper a survey of type-I ELM results is given first and recent progress is then described in detail. In between ELMs, strong mode activity is observed in a wide mode number and frequency range, specifically large amplitude $(\sim 20 \%)$ low frequency (several $\mathrm{kHz}$ ) fluctuations. The initial dynamic ELM phase is dominated by the rapid growth of helical, low mode number structures rotating in the pedestal ExB direction, while the subsequent saturation and profile erosion phase is more complex and scenario dependent. Bursts of filaments ejected from the hot edge into the scrape-off layer are correlated with primary pedestal mode rotation. After partial edge profile collapse, a quiescent recovery phase is obtained despite substantial residual edge gradients. Pellet induced ELMs behave similarly to spontaneous ones, at least for the smallest pellets available so far.
\end{abstract}

\section{Introduction}

In present day high-confinement (H-mode) divertor tokamak operation, a significant part of plasma-wall interaction is due to edge localized modes (ELMs), releasing a substantial amount of particles and energy to the first wall and divertor in a burst-like fashion. Simple empirical extrapolation of the most dangerous type-I ELMs to the forthcoming ITER indicates possibly intolerable power load and material erosion on divertor targets and even on main chamber wall elements [1]. A more quantitative assessment requires a sound experimental data basis for model validation and extrapolation, and, to be prepared for the unfavorable case, the development of tools for ELM control e.g. via external ELM pacing. The latter must include the characterization of the externally induced ELMs in comparison to spontaneous ones. Since ELMs consist of a burst of extremely fast three-dimensional, highly non-linear helical instabilities, a detailed analysis of the ELM structure, evolution and resulting wall load is a crucial ingredient for physical understanding and extrapolation to future devices. Experimental (and related theoretical) study of ELM structure has thus been given high priority on all major divertor tokamaks (see e.g. [2] for a recent multi-machine survey of type-I ELM dynamics measurements and references therein).

In this paper, which is a based on a regular presentation to the Chengdu Fusion Energy Conference [3], we focus on contributions from the poloidal divertor tokamak ASDEX Upgrade (major radius $\mathrm{R}=1.65 \mathrm{~m}$, minor radius $\mathrm{a}=0.5 \mathrm{~m}$, vertical elongation $\mathrm{b} / \mathrm{a} \sim 1.6$, triangularity up to 0.45 ) with emphasis on the combined analysis of results from many 
different diagnostics. Specifically, exploiting the extensive set of fast edge diagnostics ASDEX Upgrade, a large amount of information has been and is being accumulated on global ELM characteristics as well as on detailed ELM structures (see e.g. [4-8] and references therein). In addition, several techniques for ELM pacing and mitigation have been tested, the most successful one relying on quasi-continuous cryogenic pellet injection [9]. The latter has even been routinely applied in radiative edge discharge scenarios to enforce regular ELMs, preventing impurity penetration and accumulation in between ELMs [10]. Starting from this large, though partly incoherent and scattered data base, an attempt is made in this paper to analyse the detailed type-I ELM evolution in time and space by combining results from different diagnostics wherever possible, and with the routinely available fast magnetic pick-up coil arrays providing a key link between various observations. Complementary to statistical analysis of many ELMs, which tends to smooth away structural peculiarities, our basic idea is to characterize a small number of 'typical' ELMs (and their variability across different shot scenarios) in detail, and to extract from that the basic mechanisms and structures involved. Since an ELM affects the whole edge in a transient, quasi-periodic fashion, the analysis must include the complete ELM cycle and the whole ELM affected edge region from inside the $\mathrm{H}$-mode barrier out to the first wall and divertor.

In the following, a survey of fast ELM related diagnostics and corresponding results is given first. Recent progress in type-I ELM analysis is then described in more detail. The spatiotemporal ELM evolution resulting from this combined database is finally discussed in comparison with presently available, still rudimentary theoretical models.

\section{Survey of ELM related diagnostics and results}

As mentioned above, the main goal of this paper is to characterize the structure and dynamics of type-I ELMs by combining the information obtained from various diagnostics into one physical picture. Accordingly, in this section, we start with a survey of available diagnostics and ELM related results obtained during previous ASDEX Upgrade experimental campaigns, partly published already in another context. New results and recent advances in existing data analysis are then described in subsequent sections. In figure 1, a poloidal ASDEX Upgrade cross section is shown together with the approximate positions of a selection of fast edge diagnostics. The most important diagnostic features and related results are sketched in the following, roughly grouped with respect to the spatial region to which they contribute.

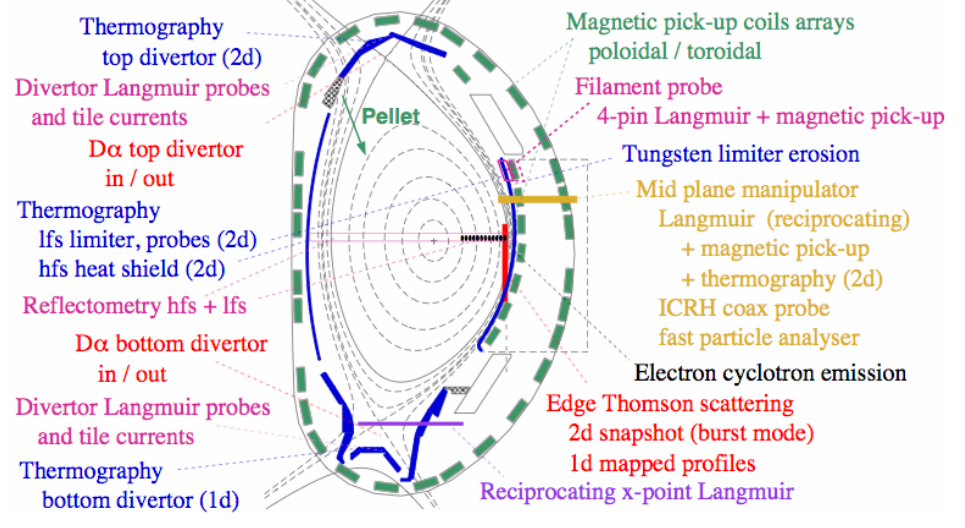

FIG.1. Poloidal cross section of ASDEX Upgrade together with an upper single null equilibrium, the positions of a selection of fast ELM related diagnostics and the pellet injection path indicated. 


\subsection{ELM effects in the hot plasma edge near to and inside the separatrix}

Since ELMs are highly non-linear electromagnetic events originating mainly from the transport barrier region just inside the separatrix, magnetic pick-up coil arrays are prime candidates for ELM structure analysis. In fact, fast magnetic probes have been and are widely used to investigate coherent precursor modes, to monitor ELM onset and duration etc. However, the detailed structural information contained in the transient probe signals during the ELM burst and semi-coherent inter-ELM events, has not yet been sufficiently exploited. Therefore, a major part of this paper will be devoted to recent progress in magnetic probe analysis; see section 3. The disadvantage of magnetic probes is that, without additional information, the radial location of the responsible perturbations remains uncertain. Therefore, magnetic probe measurements are complemented by local edge plasma diagnostics. In the hot edge plasma, inaccessible to material probes, these are mainly Thomson scattering, electron cyclotron emission and reflectometry. The latter has provided e.g. the density profile evolution during the ELM cycle [11], the fast density rise onset after pellet injection [12], and the evolution of the radial edge electric field via Doppler shifted microwave reflection on rotating high-n modes $[13,14]$. Edge Thomson scattering, run in $2 \mathrm{~d}$ snapshot mode, has revealed large amplitude 'holes' in the pedestal and 'blobs' in the hot SOL plasma during ELMs, and also in between $[15,16]$. A recent statistical analysis of such perturbations and a comparison with fluctuations seen simultaneously on the edge electron cyclotron emission (ECE) from the pedestal will be presented in section 4.

The active lithium beam diagnostic has provided inter-ELM density and ion temperature profiles [17] in the barrier and hot SOL, but was usually too slow to reveal ELM substructures. In principle, also other spectroscopic measurements and soft-X radiation might be useful, but were not available with sufficient edge resolution during past campaigns.

\subsection{Scrape-off layer (SOL) filaments induced by ELMs and inter-ELM events}

In addition to non-perturbative diagnostics like Thomson scattering (see above), the low power SOL wing is directly accessible to material probes. In this context, a key instrument on ASDEX Upgrade is the reciprocating low field side (LFS) manipulator, which can be used to expose probes of various kinds at a specific radius for a pre-defined time interval or to quickly scan radial profiles. Equipped with a multi-pin Langmuir probe head and/or magnetic pick-up coils, it has been widely used to investigate the ELM induced filament structures and dynamics in the scrape-off layer wing and limiter vicinity $[18,19]$. Recently, a specific in-outswitchable 'filament probe' with several Langmuir pins and one magnetic probe incorporated has been implemented near the manipulator on approximately the same magnetic flux bundle $[20,21]$. These local probe measurements have been complemented by a $2 \mathrm{~d}$ thermography camera measuring the power flux onto their casings (and to nearby limiters; see below). More details on the measured filament rate, size, velocities, radial decay and their correlation with primary pedestal modes will be given in section 5 .

In addition to these main chamber probes, a reciprocating divertor Langmuir probe with three pins in a Mach-type arrangement has been used to scan the lower divertor plasma horizontally near to or below the lower x-point. Transient divertor flow reversal during the ELM cycle has been routinely observed [22]. A particularly striking result was a sort of ELM size mitigation (transition to small high frequency ELMs similar to type-I to type-III transition) in H-mode plasmas with heating just above the $\mathrm{L}-\mathrm{H}$ threshold, happening whenever the probe pin (not the probe body) was close to one of the two divertor separatrix 
legs [23]. It is surprising that the small localized current drawn (few amperes) and/or the related bias voltage have such a remarkable effect on pedestal stability and ELM release.

First $2 \mathrm{~d}$ fast camera movies have shown rotating helical, field aligned filaments [24]. Filaments during and in between ELMs have been also detected in the limiter shadow by Langmuir probes [25] and an ion cyclotron arcing probe [26] mounted consecutively on the mid plane manipulator. This indicates again the presence of significant inter-ELM edge activity, sometimes even releasing filaments into the SOL during this apparently quiescent phase.

\subsection{ELM footprints on LFS limiters and divertor targets}

$2 \mathrm{~d}$ thermography cameras have been used for some time to investigate ELM footprints on wall elements. Starting with the ELM power load onto divertor wall elements, a key result was the discovery of non-axisymmetric spiral structures on divertor target plates, interpreted as footprints of detached helical filaments in the SOL [27,28]. Toroidal quasi-mode numbers (average number of helical structures on a toroidal circumference) of about 8-24 were derived, with the low values preferentially in the initial ELM phase. The transient ELM power load pattern on LFS limiters gave similar quasi-mode numbers as seen on divertor targets [19]. These detailed local power load results have been supplemented by a global ELM power balance [19].

Consistent with the thermographically measured transient target load pattern, fast fluctuations $(<25 \mu \mathrm{s})$ have been observed on flush mounted divertor target probes, formally operated in ion saturation mode with an externally applied voltage of about $40 \mathrm{~V}$ [29]. The observed transient reversal of the actually measured pin current has been attributed to large, fast excursions in the plasma floating potential, transiently exceeding the probe bias voltage.

Since ASDEX Upgrade is evolving into a tungsten dominated experiment, the contribution of ELMs to first wall erosion has been investigated. Fast spectroscopic tungsten influx measurements at low field side limiters indicate significantly enhanced W-sputter yield during ELMs [30]. This reflects an increased mean energy of ions hitting the W-surface, which is probably due to an increase in far SOL temperature as well as an increase in fast ion load onto the limiters from neutral injection heating. The latter is supported by ELM enhanced fast ion losses in the respective energy range as measured by a high-resolution fast ion loss detector in the limiter shadow (once again sitting on the mid plane manipulator during dedicated discharges) [31].

\subsection{Pellet triggered ELM analysis}

Though the diagnosis of ELMs, triggered by injection of cryogenic deuterium pellets, is in principle the same as for spontaneous ones, a few additional measurements are required to characterize the pellet seed perturbation. Specifically, dedicated pellet ablation $\mathrm{D}_{\alpha}$-monitor signals and a set of fast $2 \mathrm{~d}$ video cameras has been applied to separate the spatio-temporal evolution of the ablation plasmoid from the ELM activity triggered by it [32]. Continuous ELM pacing $[9,10]$ as well as perturbative ELM trigger studies (with pellet injection rate $f_{\text {pellet }}$ much smaller than spontaneous ELM frequency $\left.f_{E L M}\right)[33,34]$ have been done with different sizes and velocities. In the former case, pellet injection is primarily aimed at ELM 
mitigation, while in the latter case it may be regarded as a tool to probe pedestal stability. Recent progress in detailed pellet ELM analysis will be presented mainly in section 3.2.3.

\section{ELM structure analysis by magnetic probes}

\subsection{Magnetic pick-up coil arrays and structural analysis}

In ASDEX Upgrade, there are two types of magnetic pick-up coils and coil arrays, each with $2 \mathrm{MHz}$ sampling, measuring essentially the time derivative of the radial magnetic field, $\mathrm{dB}_{\mathrm{r}} / \mathrm{dt}$ (LFS 'ballooning' or 'BAL' probes), and of the poloidal one, $\mathrm{dB}_{\theta} / \mathrm{dt}$ ('Mirnov coils'), respectively. They allow the determination of the spatial ELM structure up to intermediate toroidal mode numbers, typically $\mathrm{n}_{\text {tor }}<10$. A third poloidal BAL-array with double spatial resolution (twice the upper mode number-limit) and $2.5 \mathrm{MHz}$ sampling is focused on frequencies above $50 \mathrm{kHz}$ and limited to a $400 \mathrm{~ms}$ interval per discharge. While there are densely spaced poloidal probe arrays in several sectors, the toroidal probe distribution is more scarce and inhomogeneous. This is fully adequate for coherent, rotating core MHD modes, but problematic in case of incoherent edge structures with short spatial and temporal correlation length as observed during ELMs. On the other hand, as for unstable core eigenmodes, practically all macroscopic edge instabilities relevant in the present context must be approximately magnetic field aligned over their parallel wave or correlation length. This provides a structural mapping between poloidal and toroidal probe array signals, which has been repeatedly checked and confirmed for edge structures with intermediate coherence length, and will be assumed to be valid in all cases with unfavourable probe location relative to the actual mode. All shots presented in the following have standard ASDEX Upgrade magnetic field and plasma current polarity: seen from top, the toroidal magnetic field vector is clockwise (indicated by a negative toroidal magnetic field strength in the respective shot parameter lists), while the toroidal plasma current is counter-clockwise (indicated by positive toroidal current values). For this standard setting, magnetic field lines, and consequently also field aligned edge plasma structures (with nearly vanishing parallel wave number, $\mathrm{k} / / \sim 0$ ), form a left-handed screw. If such field aligned structures rotate poloidally and/or toroidally, only the cross field phase velocity can be accurately derived from the magnetic probe arrays, leaving the parallel component essentially undetermined. Important information for comparison with theory is the direction of mode rotation in real space and relative to the ion and electron diamagnetic drift directions. In this paper, these velocities will be mostly represented by their poloidal LFS component using "upward" and "downward" for motion in positive and negative z-direction, respectively. For the standard magnetic field polarity outlined above, the electron diamagnetic drift velocity is upward and the ion drift velocity downward.

Based on these preliminaries, and in order to allow for easy direct comparison between different ELM examples, the following presentation of probe results will be largely focused on signals from a specific LFS poloidal probe array located in sector 15, complemented by one high field side (HFS) probe, the pellet ablation monitor and the familiar in/out divertor $\mathrm{D}_{\alpha}$ signals as ELM monitors (see insert top/right in fig.2). Local poloidal mode numbers and phase velocities derived from the LFS array are then approximately converted to toroidal values by simply using the local LFS magnetic field line pitch angle. The latter is typically $\mathrm{B}_{\text {pol }} / \mathrm{B}_{\text {tor }} \sim 1 / 5$ (depending on $\mathrm{q}_{95}$, plasma shape, etc), with slow poloidal variation and rather weak radial variation even across the separatrix., i.e. there is only a small local LFS field line 


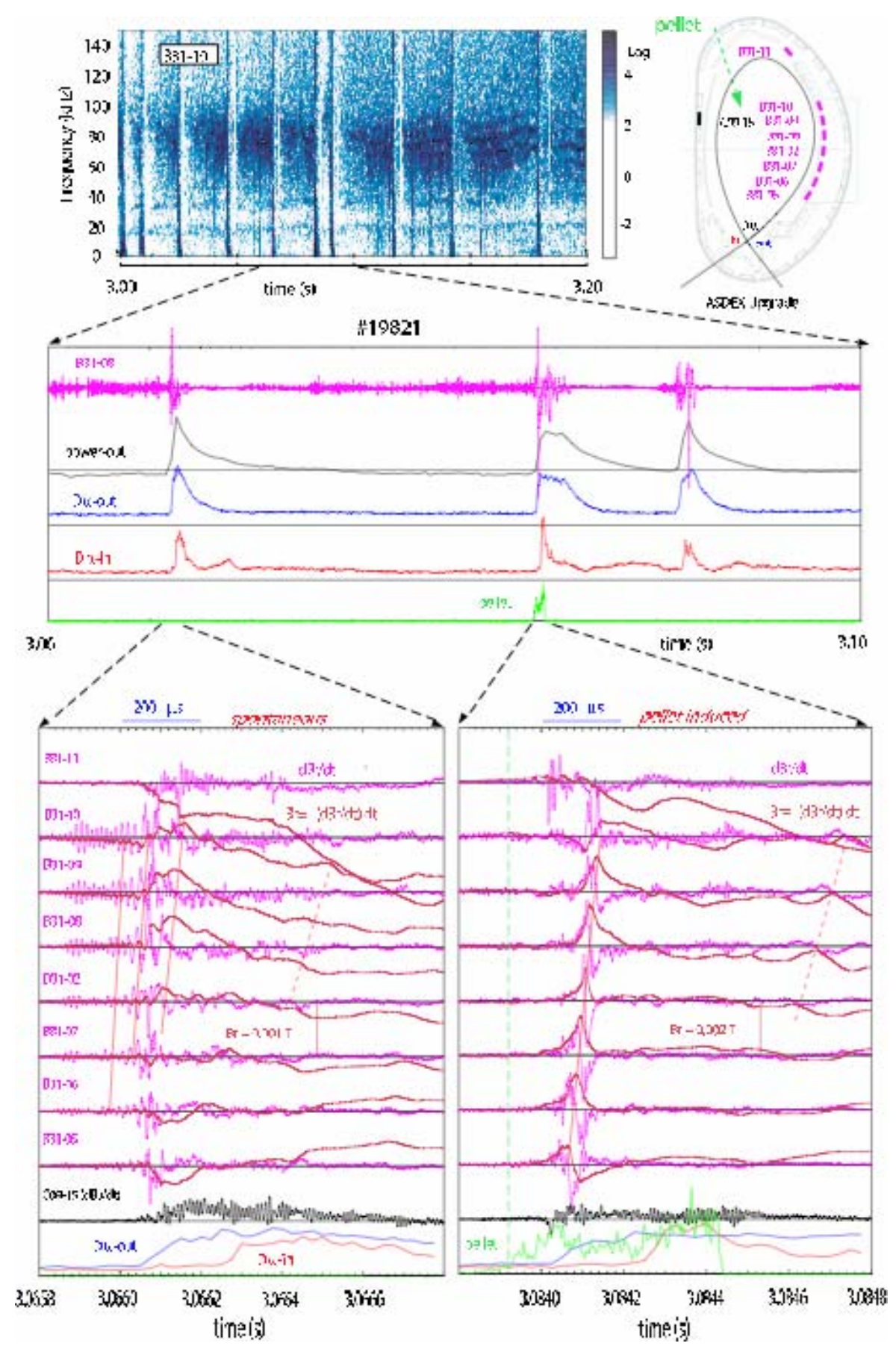

FIG. 2. Top: Spectrogram of a selected magnetic pick-up coil of shot \#19821, together with an insert indicating probe positions, the pellet injection path and the separatrix shape at $t=3.06 \mathrm{~s}$.

Mid: Time traces of a magnetic probe, power to outer target, inner/outer divertor $D_{\alpha}$ and pellet monitor for the selected spontaneous and pellet triggered ELMs (arbitrary units).

Bottom: The rapidly varying $d B(t) / d t$ signals are shown together with their much smoother integrals $B(t)$ for eight low field side and one high field side probe in comparison with inner/outer divertor $D_{\alpha}$ and the pellet ablation monitor (left: spontaneous ELM, right: pellet triggered ELM). Since the time traces are vertically staggered at equal distances, as are the probes B31-05 to B31-10 in real space (see insert top/right), the poloidal velocity and width of moving structures can be read directly from the plot (inclined vertical lines). 
shear, in contrast to the global one exhibiting a singularity at the separatrix. Finally, one should also notice that in contrast to coherent eigenmodes, $\mathrm{n}_{\text {tor, }}$ if used as a quasi-mode number to characterize transient, more or less irregular structures as done in this paper, is not necessarily an integer.

\subsection{Spontaneous versus pellet induced ELM: a 'typical' pair}

\subsubsection{Discharge scenario and inter-ELM activity}

As a first example, we discuss a 'typical' spontaneous-versus-induced ELM pair selected from shot \#19821, a discharge tailored for perturbative ELM pacing analysis. A list of scalar parameters, both global and pedestal-top, for this and the other discharges considered in this paper is given in table 1. The basic idea behind the choice of shot \#19821 is to compare fully developed, well separated type-I ELMs of both kinds (and their precursors) under roughly identical start conditions and with as little as possible confusion by ELM-irrelevant perturbations like core MHD modes. Before entering the detailed ELM analysis, we give an overview of the selected discharge phase, including inter-ELM mode activity and precursor modes of possible relevance for ELM onset.

Figure 2 (top) shows a frequency spectrogram of $\mathrm{dB}_{\mathrm{r}} / \mathrm{dt}$ measured by the BAL probe 31-10, which is closest to the actual LFS plasma surface (sliding FFT of 2048 points, equivalent to $\sim 1 \mathrm{~ms}$, using a Hanning window and $75 \%$ window overlap; no frequency smoothing). A $200 \mathrm{~ms}$ discharge window is covered, containing several spontaneous ELMs (appearing as broadband vertical lines), and one ELM triggered by a single pellet injected at $t=3.084 \mathrm{~s}$. The average pedestal top electron temperature and density in this window are $\mathrm{T}_{\mathrm{e}, \mathrm{ped}} \sim 530 \mathrm{eV}$ and $\mathrm{n}_{\mathrm{e} \text {,ped }} \sim 4.510^{19} \mathrm{~m}^{-3}$. The resulting normalized electron pedestal collisionality is $v^{*}{ }_{\text {ped }} \sim$ 0.6. (For comparison, the hoped-for $v^{*}$ ped in ITER is $\sim 0.07$.) The ion temperature $T_{i, p e d}$, not measured in this discharge, is usually slightly higher for such a shot [8], yielding a correspondingly lower ion collisionality. In between ELMs there is a high level of broadband noise, several mini-events (weak vertical lines) and especially some quasi-coherent mode activity between about 60 and $100 \mathrm{kHz}$, growing shortly after an ELM and lasting until the next one. There appear to be several, roughly equidistant, semi-coherent frequency bands, similar to harmonics of a slowly varying base frequency $\mathrm{f}_{\mathrm{b}}$ of about $10 \mathrm{kHz}$, with maximum mode amplitude around a specific harmonic, e.g. somewhere around $\mathrm{n}_{\mathrm{h}} \sim 8$. Qualitatively, these modes are reminiscent of the washboard modes observed in JET [35,36]. The probe signal itself (time domain) shows a fluctuating, amplitude modulated wave train, with more or less discrete frequency transitions between these high harmonics as indicated by the detailed spectrogram pattern. Poloidal and toroidal magnetic probe analysis reveals a helical, field aligned mode structure with toroidal mode number around $\mathrm{n}_{\text {tor }} \sim 8$, rotating poloidally upward at LFS (electron drift direction) with $\mathrm{v}_{\text {pol }} \sim 20 \mathrm{~km} / \mathrm{s}$ or, equivalently, in counter current toroidal direction at about $100 \mathrm{~km} / \mathrm{s}$. Notice that, because of approximately constant phase along field lines, this poloidal velocity is essentially equal to the cross field phase velocity component of the mode. Assuming that such macroscopic MHD-type modes (local poloidal wavelength at LFS about $0.3 \mathrm{~m}$ ) rotate with the ExB velocity, we get an inward directed radial electric field in the laboratory frame of about $40 \mathrm{kV} / \mathrm{m}$, consistent with typical $\mathrm{H}$-mode edge barrier values $[6,14]$. This is a first hint, though no explicit proof, that these intermediate-n modes are radially located in the steep gradient edge, as they are in JET [36]. 
Table 1: Scalar Parameters of the Shots Analyzed

\begin{tabular}{|l|c|c|c|c|c|c|c|c|}
\hline Shot Number & 19821 & 20176 & 20040 & 20054 & 21015 & 19807 & 20419 & 21292 \\
\hline time [s] & 3.1 & 4.5 & 4.5 & 4.5 & 4.5 & 2.6 & 4.5 & 2.2 \\
\hline Configuration & LSN & LSN & LSN & LSN & LSN & LSN & LSN & LSN \\
\hline Ip [MA] & 1.0 & 0.8 & 1.0 & 1.0 & 1.0 & 1.0 & 1.0 & 0.8 \\
\hline Bt [T] & -2.7 & -2.1 & -2.7 & -2.7 & -2.0 & -2.5 & -2.7 & -3.0 \\
\hline$q_{95}$ & 4.8 & 4.7 & 5.0 & 5.2 & 3.8 & 4.6 & 5.3 & 6.1 \\
\hline R [m] & 1.63 & 1.63 & 1.62 & 1.62 & 1.61 & 1.62 & 1.62 & 1.64 \\
\hline a [m] & 0.49 & 0.46 & 0.50 & 0.49 & 0.50 & 0.50 & 0.50 & 0.48 \\
\hline$\beta_{\mathrm{N}}$ & 1.0 & 3.0 & 1.5 & 1.6 & 2.2 & 1.3 & 2.1 & 1.4 \\
\hline$\beta_{\text {pol }}$ & 0.57 & 1.88 & 0.91 & 0.95 & 1.12 & 0.72 & 1.32 & 1.08 \\
\hline li & 0.98 & 0.92 & 1.11 & 1.11 & 1.06 & 0.98 & 1.04 & 1.09 \\
\hline$\kappa($ separatrix) & 1.78 & 1.80 & 1.73 & 1.76 & 1.78 & 1.71 & 1.75 & 1.64 \\
\hline$\delta($ separatrix) & 0.21 & 0.25 & 0.24 & 0.24 & 0.23 & 0.21 & 0.25 & 0.24 \\
\hline Lower triangularity & 0.37 & 0.44 & 0.37 & 0.38 & 0.34 & 0.39 & 0.38 & 0.43 \\
\hline Upper triangularity & 0.05 & 0.07 & 0.10 & 0.10 & 0.13 & 0.13 & 0.13 & 0.05 \\
\hline ne $\left[10^{19}\right.$ m $\left.^{-3}\right]$ & 5.8 & 5.9 & 6.6 & 5.9 & 10.0 & 9.3 & 7.2 & 5.2 \\
\hline Input power [MW] & 5.2 & 12.4 & 6.4 & 6.4 & 7.2 & 8.0 & 11.8 & 6.7 \\
\hline Te,ped [eV] & 530 & 730 & 570 & 730 & 290 & 520 & 650 & $\mathrm{n} / \mathrm{a}$ \\
\hline ne,ped [10 $\left.{ }^{19} \mathrm{~m}^{-3}\right]$ & 4.5 & 4.5 & 5.4 & 4.3 & 8.2 & 5.2 & 6.3 & 5.6 \\
\hline${ }^{*}$, ped & 0.6 & 0.4 & 0.7 & 0.3 & 2.9 & 0.7 & 0.6 & $\mathrm{n} / \mathrm{a}$ \\
\hline
\end{tabular}

The equidistant washboard-type mode pattern seen on the spectrogram might have a simple and useful physical explanation: Assume that modes of the same kind, but with different amplitude and different integer wave numbers $\mathrm{n}_{\text {tor }}$, grow and decay in parallel or sequentially with radial eigenfunctions peaked around the same rational q-surface. These modes are expected to rotate with about equal velocity and therefore a fixed magnetic pick-up coil would simply detect equidistant harmonics $f_{h}$ proportional to respective toroidal wave numbers $n_{\text {tor }}$, with a common base frequency $f_{b}$ as multiplier, $f_{h}=n_{\text {tor }} f_{b}$, and with $f_{b}$ simply given by the toroidal rotation frequency of a fictitious $\mathrm{n}_{\text {tor }}=1$ mode. Applied to the single probe spectrogram in figure 2, this picture yields roughly $\mathrm{f}_{\mathrm{b}} \sim 10 \mathrm{kHz}$ consistent with the toroidal phase velocity and the torus dimensions given earlier. The fact that the most intense harmonics are located in the vicinity of $\mathrm{n}_{\text {tor }}=8$ is also consistent with the approximate wave number, $\mathrm{n}_{\text {tor }} \sim 8$, derived independently by a much more elaborate combined structural analysis of many probe signals. If this picture applies, then the most unstable modes and their wave number can be directly read off and compared with theoretical stability diagrams of e.g. internal peeling-ballooning modes.

The spectrogram in figure 2 indicates also a semi-coherent mode near $20 \mathrm{kHz}$ (and its first harmonic). According to LFS probe array signals, this is a $\mathrm{n} \sim 3$ mode rotating downward, i.e. in the ion drift direction, with $\geq 10 \mathrm{~km} / \mathrm{s}$. Following the same hypothesis, this would correspond to an outward radial electric field of order $20 \mathrm{kV} / \mathrm{m}$. Such values are typically measured by Doppler reflectometry (based on very high-n turbulent mode rotation measurement) in H-mode well inside the barrier [14], but still within the ELM affected area, e.g. near $\mathrm{q}=3$. For comparison, in $\# 19821$ the $\mathrm{q}=5$ surface is located in the steep gradient region, $\mathrm{q}=4$ near pedestal top, and $\mathrm{q}=3$ and $\mathrm{q}=2$ about $4 \mathrm{~cm}$ and $15 \mathrm{~cm}$ inside the LFS separatrix, respectively. In contrast to the short wavelength washboard-type modes, these lower-n modes are visible also on the more distant top BAL and Mirnov probes. Though again modulated by the ELM cycle, the lower-n modes are obviously not causal for ELMs, since ELMs occur also in their absence. 
Next, in figure 2 (mid), we zoom into the discharge phase containing the selected spontaneous and pellet triggered ELMs. Though qualitatively similar, there are a few obvious differences, specifically the longer pulse duration of the promptly triggered ELM compared to the spontaneous one. The detailed magnetic signature of this ELM pair is presented in the bottom part of figure 2 . Time traces are shown over a $1 \mathrm{~ms}$ window each, on the right side for the pellet triggered ELM and on the left for the preceding spontaneous ELM. The magenta lines show the rapidly varying $\mathrm{dB}_{\mathrm{r}} / \mathrm{dt}(\mathrm{t})$ signals from the LFS poloidal BAL array (inset figure 2 (top/right)) and the red-orange ones give the their much smoother integrals $B_{r}(t)$ (with $\mathrm{B}_{\mathrm{r}}$ set to zero at the window start; absolute $\mathrm{B}_{\mathrm{r}}$-scale indicated). Since the time traces are vertically staggered at equal distances, as are the probes B31-05 to B31-10 in real space, the poloidal velocity and width of moving structures can be read directly from the plot. In addition, one high field side Mirnov coil signal $\left(\mathrm{dB}_{\theta} / \mathrm{dt}\right)$, and the $\mathrm{D}_{\alpha}$ emission from inner/outer divertor and from the ablating pellet, if present, are shown (see figure 2 top/right for poloidal diagnostic locations and the pellet path).

\subsubsection{Spontaneous ELM}

Focusing on the spontaneous ELM traces first, we see initially ( $\mathrm{t} \leq 3.0660 \mathrm{~s})$ the above discussed washboard-type precursor mode with maximum amplitude above mid plane, where the equilibrium is closest to the probe array (radial mode decay length about $5 \mathrm{~cm}$ for $\mathrm{n}=8$ ). Notice that this mode is nearly invisible on the top and HFS probes. This might indicate a dominant LFS ballooning structure. Since, however, the poloidal wavelength on the HFS $(\geq$ $10 \mathrm{~cm}$ ) is nearly a factor of three smaller than on LFS, the sensitivity of the distant HFS Mirnov probes is also much smaller. This upward rotating precursor mode $\left(\mathrm{v}_{\mathrm{pol}}>20 \mathrm{~km} / \mathrm{s}\right)$ appears to grow and slow down as indicated by the inclined vertical lines of constant phase (figure 2, bottom), and to form the dominant ELM structure in this very first ELM phase around the $\mathrm{dB} / \mathrm{dt}$ maximum $(\mathrm{t} \leq 3.0661 \mathrm{~s})$. In parallel, the outer divertor $\mathrm{D}_{\alpha}$ and the power load onto divertor targets (from thermography; figure 2, mid) start to rise, indicating progressive edge erosion and transport into the SOL and divertor. The time integrated $\mathrm{B}_{\mathrm{r}}(\mathrm{t})$ traces, however, indicate a significant increase of the poloidal wavelength, equivalent to decreasing quasi-mode number, roughly $\mathrm{n}_{\text {tor }} \sim 4$ (estimated according to section 3.1 ). This means that either the initial washboard-type mode (possibly localized on a rational q-surface as argued above) might be non-linearly transformed into another one, or that a similar, more global mode becomes rapidly unstable and quickly dominates over the precursor mode. Additional high frequency components appear simultaneously on the LFS (e.g. $\sim 140 \mathrm{kHz}$ ) and on HFS dB/dt (e.g. $\sim 90 \mathrm{kHz}$ ), but are smoothed away on $\mathrm{B}(\mathrm{t})$. Altogether, this initial highly dynamic ELM phase up to the $\mathrm{B}_{\mathrm{r}}$ maximum lasts for less than $200 \mu \mathrm{s}$. In this context, one should notice that it is $\mathrm{B}_{\mathrm{r}}$ which measures the radial mode excursion. Assuming a parallel wave number $\mathrm{k} / / \sim 1 /(\mathrm{qR})$ as expected for LFS ballooning modes, together with the experimental maximum of $\mathrm{B}_{\mathrm{r}} / \mathrm{B}_{\text {tor }} \sim 10^{-3}$ (extrapolated to the pedestal radius), we estimate a radial excursion during the ELM of $\sim 5 \mathrm{~mm}$ (with large error margins), i.e. of order of the pressure decay length at the position of maximal gradient. The $\mathrm{B}_{\mathrm{r}}(\mathrm{t})$ modulation over the next few $100 \mu$ s and beyond is partly due to the usual ELM induced inward $n=0$ equilibrium shift, reaching $3 \mathrm{~mm}$ at the end of the time window. However, the toroidally distributed, nonequidistant mid plane probes indicate also significant toroidal variations. Still upward rotating structures can be identified, as the one indicated by an inclined dashed line $(\mathrm{t}$ $3.0665 \mathrm{~s}$ ), corresponding to $\mathrm{v}_{\mathrm{pol}} \sim 4 \mathrm{~km} / \mathrm{s}$. Following again the ExB rotation hypothesis (section 3.2.1 above), this would correspond to $E_{\mathrm{r}} \sim-8 \mathrm{kV} / \mathrm{m}$, not far above typical L-mode 
edge values of about $-5 \mathrm{kV} / \mathrm{m}[14]$.

In passing we note that the described scenario is not unique for this specific ELM, but is qualitatively recovered for all spontaneous ones contained in and near the chosen time window. This applies also for similar discharges with moderate heating and gas puffing, but the inter-ELM and precursor activity varies significantly. Frequently, the discrete washboardtype spectral lines appear to be absent, though there still remains some broadband high frequency turbulence. An example with apparently exponential ELM growth out of low amplitude precursor noise will be given below (section 3.3).

The most prominent features described above are also recovered for rather different type-I ELM regimes with much higher heating power, e.g. \#20176, an improved H-mode discharge (see Table 1 for the discharge parameters). However, the appearance of additional modes, especially core modes masking the edge effects, makes these scenarios unattractive for basic ELM physics studies.

\subsubsection{Pellet induced ELM}

The magnetic signature of a pellet induced ELM is given in figure 2 (bottom/right). With a particle content of about $510^{19} \mathrm{D}$-atoms at $240 \mathrm{~m} / \mathrm{s}$, the injected pellet is near the lower injector mass limit, but still penetrates well beyond the pedestal, depositing particles all along its path. Looking onto the top spectrogram in figure 2, there is no visible difference between both ELMs, except for the fact that the pellet ELM is longer $\left(\sim 2 \mathrm{~ms} \mathrm{D}_{\alpha}\right.$ pulse) and quickly followed by a second one, with clearly different precursor structure in between. Looking at the magnetic signals in comparison with the pellet ablation trace, we recover the familiar pellet trigger sequence [34,37]: The prompt ELM is triggered already when the pellet crosses the barrier region, and those particles deposited in the barrier region have a good chance to be quickly expelled again by the ELM. In contrast, the pellet mass deposited deep inside the barrier returns to the edge only on a radial transport time of several ms (in parallel to radial heat transport), triggering the delayed satellite ELM via enhanced axisymmetric edge profile steepening.

The detailed magnetic fingerprint of the promptly triggered ELM exhibits a few distinct differences with respect to the spontaneous one. The maximum $B_{r}$ values are about a factor of two higher (factor 2 different $\mathrm{B}_{\mathrm{r}}$ scale as indicated), there is no strong precursor mode in the sense of a mode growing out of the inter-ELM fluctuations as observed for the spontaneous ELM, and the top and inside probes start earlier than the LFS probes. The latter is most probably due to the top-inside pellet seed perturbation, a helical plasmoid sheath, expanding along field lines and initiating the ELM [33]. On the probe array, we see initially a rather clear singular helical structure travelling once more upward at similar speed as observed for the spontaneous ELM $(\sim 10 \mathrm{~km} / \mathrm{s})$. Several slower upward rotating structures with decreasing amplitude can be identified over the whole duration of the slightly modulated $D_{\alpha}$ pulse (partially outside the zoomed window; see figure2, mid). One such structure is indicated by the inclined dashed line $(\mathrm{t} \sim 3.0847 \mathrm{~s})$, corresponding to $\mathrm{v}_{\mathrm{pol}} \sim 4 \mathrm{~km} / \mathrm{s}$, the same value as observed in the spontaneous ELM after the profile crash. The poloidal structure as well as the temporal sequence indicates a low toroidal quasi-mode number near $\mathrm{n}_{\text {tor }} \sim 3$. The high frequency mode seen simultaneously in the ELM decay phase $(\sim 120 \mathrm{kHz}, \mathrm{n} \sim 4)$ moves rapidly downward, i.e. in ion drift direction, but its radial location remains to be determined. Though obviously triggered by the ELM, it may be located inboard of the edge barrier, but still in the ELM affected area. 
Another 15 pellet induced ELMs in similar discharges (\#20040, \#20054) have been analysed for their basic poloidal/toroidal structure. It has been found that the single, upward rotating structure seen in figure 2 , bottom/right accidentally represents a rather specific case. In the majority of cases, two or more such pronounced structures occur simultaneously, forming a sort of rotating and slowly distorting wave packet. These structures slow down by a factor of two and decay in amplitude on a few $100 \mu$ s time scale. Despite the fixed pellet launch position, they appear initially at a more or less random toroidal position relative to the pellet, i.e. they are not directly growing out of the pellet plasmoid. The remaining cases show less strong and pronounced structures and are more similar to typical spontaneous ELM signatures. The most extreme pellet ELM scenario, superficially analysed only so far, has been obtained in a stationary radiative edge discharge (\#21015) with external argon puffing and continuous pellet ELM pacing $(55 \mathrm{~Hz}, 880 \mathrm{~m} / \mathrm{s})$ to prevent a radiation collapse. Also in this case, the qualitative ELM behaviour is as above with upward rotating low-n structures at rather similar velocity during and after the barrier crash. However, in between ELMs, a narrow helical $n=1$ snake-like perturbation rotating with about $2 \mathrm{kHz}$ is seen. It appears a few ms after each ELM and may last until the next one. Since the (detached) divertor radiation is strongly modulated at the $n=1$ frequency, this mode may be located somewhere close to the separatrix. Furthermore, the long lifetime may indicate an island-type structure. It is not yet clear whether its existence is linked to pellet injection or just to enhanced edge radiation.

\subsection{Spontaneous 'seed' filament growth as ELM trigger}

We conclude this section with a spontaneous ELM from discharge \#19807, which was initiated by one helical LFS filament structure in the torus section where the poloidal magnetic probe array is located. Figure 3 shows a $0.5 \mathrm{~ms}$ zoom of the ELM initiation phase, using the same signals, colors and definitions as in figure 2 (bottom). The pedestal top electron temperature and density in \#19807 are very similar to those corresponding to the spontaneous ELM in figure 2, $\mathrm{T}_{\mathrm{e} \text {,ped }} \sim 520 \mathrm{eV}$ and $\mathrm{n}_{\mathrm{e} \text {,ped }} \sim 5.210^{19} \mathrm{~m}^{-3}$, so that $v^{*}$ ped $\sim 0.7$. There is little high frequency precursor activity, especially no pronounced washboard mode. At about $\mathrm{t}=2.6074 \mathrm{~s}$, a burst of activity is observed on the upper half array which then decays without leading to an ELM. We interpret this as a 'premature' perturbation which has, for whatever reason, not provided a sufficient perturbation to trigger the non-linear ELM crash phase. Near $t=2.6075 \mathrm{~s}$, the probe signals in the upper half array start to grow exponentially on a few $\mu$ s time scale and with roughly opposite phase on the adjacent probes B31-08 and -09 , separated by $\sim 15 \mathrm{~cm}$. The effective poloidal width is of order $\sim 60 \mathrm{~cm}$ with essentially one cross field wavelength on it (equivalent to $n_{\text {tor }} \sim 4$ ). The toroidal probe array confirms once more the magnetic field aligned geometry. This helical wavelet structure saturates at macroscopic amplitude and rotates again poloidally upward with about $20 \mathrm{~km} / \mathrm{s}$.

With about $50 \mu$ s delay, this first helical low-n LFS filament structure, grown more or less out of noise, triggers a global ELM with all magnetic probe signals on the torus rising rapidly within few $10 \mu \mathrm{s}$.

Still the question remains, why this first filament suddenly grows at a high rate after a long apparently quiescent phase. Looking again at figure 3 , we see a slow pre-ELM variation on $\mathrm{B}_{\mathrm{r}}(\mathrm{t})$, which might indicate some low-frequency variation difficult to identify on $\mathrm{dB} / \mathrm{dt}$. This question will be further addressed in the next section. 


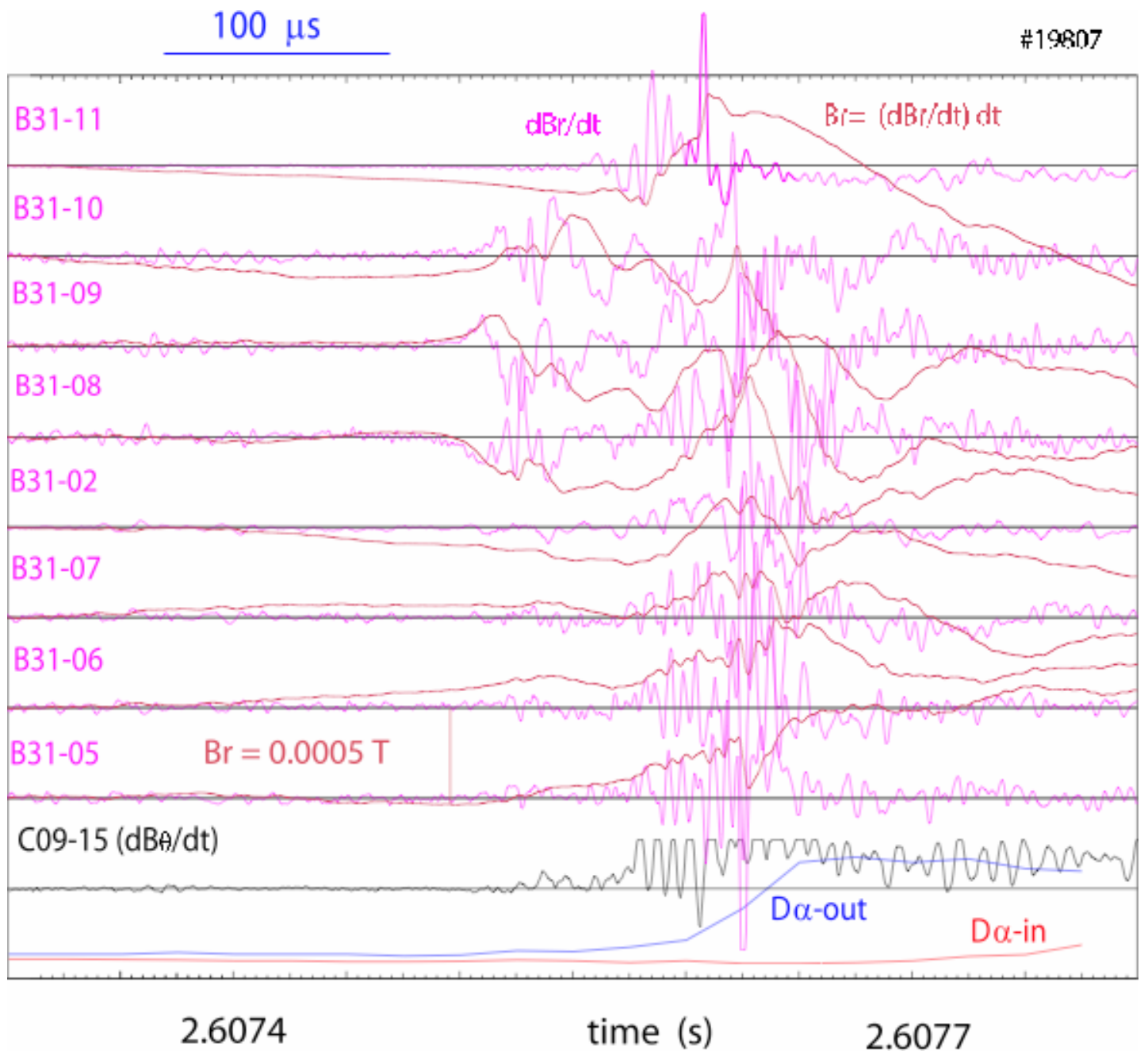

Figure 3: The rapidly varying $d B(t) / d t$ signals and their smooth integrals $B(t)$ are shown for eight low field side and one high field side probe in a $0.5 \mathrm{~ms}$ window in comparison with inner/outer divertor $D_{\alpha}$ (essentially same format as in figure 2, bottom). A premature perturbation structure at $t=2.6074 \mathrm{~s}$ and the seed filament after $t=2.6075 \mathrm{~s}$ can be identified on the upper part of the array.

\section{Edge-pedestal perturbations identified by Thomson scattering and ECE}

In standard operation, the multi-pulse edge Thomson scattering system is run in quasicontinuous mode with all lasers fired consecutively in time. Electron temperature $T_{e}$ and density $\mathrm{n}_{\mathrm{e}}$ in the respective scattering volumes are then mapped onto a $1 \mathrm{~d}$ radial coordinate. These profiles show a significant data scatter beyond statistical noise, which has been attributed to local filamentary structures appearing as outliers on an average $1 \mathrm{~d}$ profile [38]. In fact, exploiting the $2 \mathrm{~d}$ arrangement of scattering volumes, and running the radially staggered, vertical YAG laser beams in burst mode within 2.5 microseconds, $2 \mathrm{~d}$ snapshots have been constructed revealing holes in the pedestal and blobs in the scrape-off layer during ELMs, and even in between, corresponding to estimated toroidal quasi-mode numbers of $n_{\text {tor }}$ $\sim 8-20$ [15]. Apart from a slowly moving structure in the poloidal $\mathrm{B}_{\mathrm{r}}$ pattern (measured five sectors away in counter current direction), no clearly correlated magnetic signature could be 
identified, suggesting that these perturbations might be due to nearly locked modes.

In a more elaborate study, about 527 snapshots from shots \#20414-20420 have been statistically analysed in substantial detail [16]. The scalar parameters of one of these time slices are shown in table 1 . About $60 \%$ of the analysed ELMs showed sufficiently clear structures to derive an approximate toroidal quasi-mode-number, typically in the range $\mathrm{n}_{\text {tor }}=$ $6-48$ without drastic difference between ELM and inter-ELM phases. The lower n-limit is not a physical limit, but determined by the diagnostic. The relative perturbation amplitudes are mostly a few tens of percent, with a tendency for larger temperature than density modulation. Typical pedestal top parameters in this discharge set were $\mathrm{T}_{\mathrm{e} \text {,ped }} \sim 650 \mathrm{eV}$ and $\mathrm{n}_{\mathrm{e}, \text { ped }} \sim 6.310^{19} \mathrm{~m}^{-3}$, yielding a normalized collisionality $v^{*}{ }_{\text {ped }} \sim 0.6$, similar to the value reported earlier for \#19821 and \#19807. One should notice, however, that $v^{*}$ increases rapidly towards the separatrix. Radial $\mathrm{T}_{\mathrm{e}}$ and $\mathrm{n}_{\mathrm{e}}$ profiles from Thomson scattering have been included in reference [16] (figure 3) for \#20417 before, during, and after ELMs, from which parameters such as the local collisionality may be calculated.

Recently, a comparison between such $2 \mathrm{~d}$ snapshots and electron cyclotron emission (ECE; 32 $\mathrm{kHz}$ sampling; six sectors or $130^{\circ}$ away in co-current-direction) has identified discharges that clearly show repeated transient mode structures in the pedestal on the ECE for the same time interval where the $2 \mathrm{~d}$ snapshots see pedestal modes on approximately the same flux surfaces. One of these shots with lasers in burst mode and with edge optimized ECE settings is shot \#19807, used already in the previous section. Figure 4 shows an example with a particularly strong double structure seen by the Thomson diagnostic on the $2 \mathrm{~d}$ density and temperature contour plots (left). While contours of density and temperature coincide nicely with flux surfaces in the unperturbed plasma equilibrium, we find in this example two rather pronounced 'holes' near the separatrix in temperature as well as in density (see reference [16], figure 4, for more examples and technical details). Examining the $\mathrm{B}_{\mathrm{r}}(\mathrm{t})$ signals near the snapshot instant, we find a few slowly downward moving dips (around $1 \mathrm{~km} / \mathrm{s}$ ), which might be related to these pedestal holes or corresponding expelled filaments. In fact, the observed velocity and dip duration $(\sim 200 \mu \mathrm{s})$ would be consistent with the poloidal $2.5 \mu$ s snapshot pattern.

In the right part of figure 4, the ECE radiation temperature evolution from three channels in the edge transport barrier measured about six sectors $\left(\sim 130^{\circ}\right)$ away in co-current direction is presented for a $20 \mathrm{~ms}$ time window, with the Thomson snapshot time marked by a vertical line. In addition to the ELM crash, a sequence of weakly coherent events in the few $\mathrm{kHz}$ range are observed on the ECE in between ELMs in the barrier region, with a radial phase inversion over less than a centimetre. In this context, one should notice that the absolute value of the electron radiation temperature derived from ECE near the separatrix is typically larger than the electron temperature measured there by Thomson scattering and that the radiation temperature profile shows a parasitic maximum there. This effect is well known and attributed to a small non-thermal electron contribution in case of high electron temperature gradients and low electron densities, resulting in insufficient optical thickness. In consequence, near the separatrix and outside, the ECE signal may respond to density as well as temperature variations, while pedestal top temperatures should be about correct. 

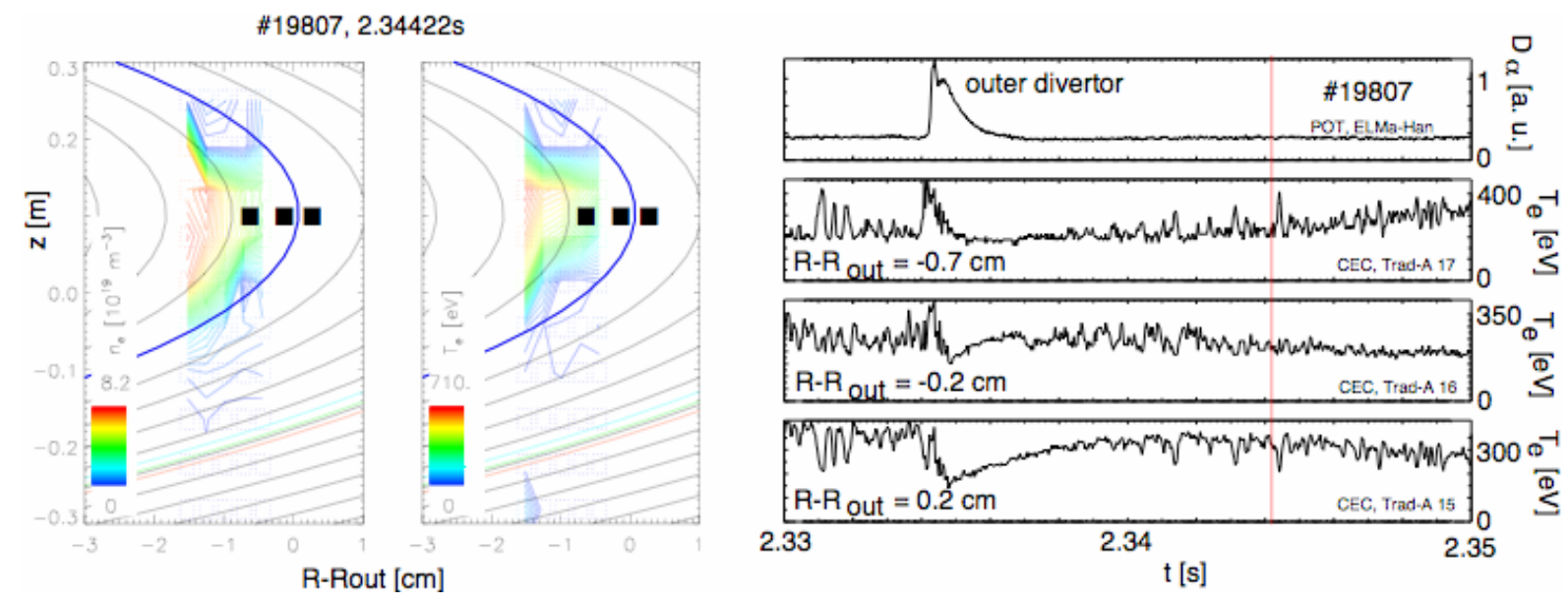

FIG. 4. Left: $2 d$ poloidal density and temperature Thomson scattering snapshots taken from shot $\# 19807$ at $t=2.34422 \mathrm{~s}$ together with magnetic equilibrium flux surface contours. The separatrix is indicated by a thick blue line (radial separatrix position error $\sim 0.5 \mathrm{~cm}$ ) and far-SOL flux surfaces touching first wall elements are marked by thin light blue, green and red lines. The rectangular, light blue dotted background matrix indicates the location of the Thomson scattering volumes (see reference [16] for more technical details). For comparison, the position of the electron cyclotron measurements (located about $130^{\circ}$ toroidally away in co-current direction) is indicated by black squares. Right: Apparent electron radiation temperature derived from electron cyclotron emission at three radii in the barrier region in comparison with divertor $D_{\alpha}$. The Thomson snapshot time is marked by a vertical line.

A spectrogram of the pedestal-top temperature (Trad-A 17) is given in the upper part of figure 5 for a discharge time window including the time interval from the previous figure (indicated by box A) as well as the ELM analysed in the previous section (box B). The FFT parameters have been adjusted to the low sampling frequency, but are otherwise as for figure 2 (64 FFT points, equivalent to $2 \mathrm{~ms}$ ). The inter ELM fluctuations are recovered as a fluctuation band, starting at a few $\mathrm{kHz}$ after an ELM and rising continuously up to $7 \mathrm{kHz}$ or so before the next ELM crash, probably due to a continuously increasing Doppler shift. If we choose somewhat arbitrarily an average toroidal mode number $\mathrm{n}_{\text {tor }} \sim 10$ according to the $\mathrm{n}_{\text {tor }}$ distribution derived from Thomson snap shots (ref. [16], figure 8), then a cross field velocity of up to a few $\mathrm{km} / \mathrm{s}$ is estimated, significantly below the ExB velocities discussed in context with the washboard-type modes. A qualitatively similar, but much less detailed low frequency fluctuation pattern in the same frequency range is indicated by the $\mathrm{B}_{\mathrm{r}}(\mathrm{t})$ spectrogram of probe B31-14 (figure 5, bottom; sliding FFT of 4096 points, again equivalent to $2 \mathrm{~ms}$ ); see e.g. ELM interval around $\mathrm{t} \sim 2.385 \mathrm{~s}$. This probe is located at the same poloidal position as B31-02 (figure 2, top/right), but only $40^{\circ}$ toroidally away from the ECE diagnostic. Notice that, in contrast to the magnetic probes with $10 \mathrm{~cm}$ lateral dimensions and similar distance from the plasma surface (see figure 2, top/right), ECE and Thomson scattering provide local values with a few $\mathrm{mm}$ radial and a few $\mathrm{cm}$ poloidal resolution. Keeping in mind these restrictions, there is a high probability that all three diagnostics see essentially the same type of modes, i.e. substantial pedestal density, temperature and pressure perturbations with several $\mathrm{kHz}$ and a wide spatial mode number spectrum. Their cross field velocity direction is still a bit uncertain, since these modes are difficult to trace on the magnetic probe array. The only hint so far comes from the $\mathrm{B}_{\mathrm{r}}$-dips mentioned above, which indicate a rather slow rotation in the cross field ion drift direction. The fact, that these modes 
definitely reside in the steep gradient region, but do not rotate with pedestal ExB velocity may suggest that they are different in nature from those discussed earlier, e.g. tearing mode type island structures. In addition, the radial electric field in the laboratory frame might approach zero and change sign near this radial position because of local cancellation of drift and fluid rotation [5]. Notice that, even if these modes would be fully locked to external error fields $\left(\mathrm{v}_{\mathrm{pol}}=0\right)$, they would be still well captured by Thomson snapshots, but difficult to isolate from the radial ECE temperature profile, and invisible on $\mathrm{dB} / \mathrm{dt}$. Altogether, the assumption of island-type perturbations with slow downward motion or mode locking would be consistent with the experimental observations, at least for this specific shot.

(A)

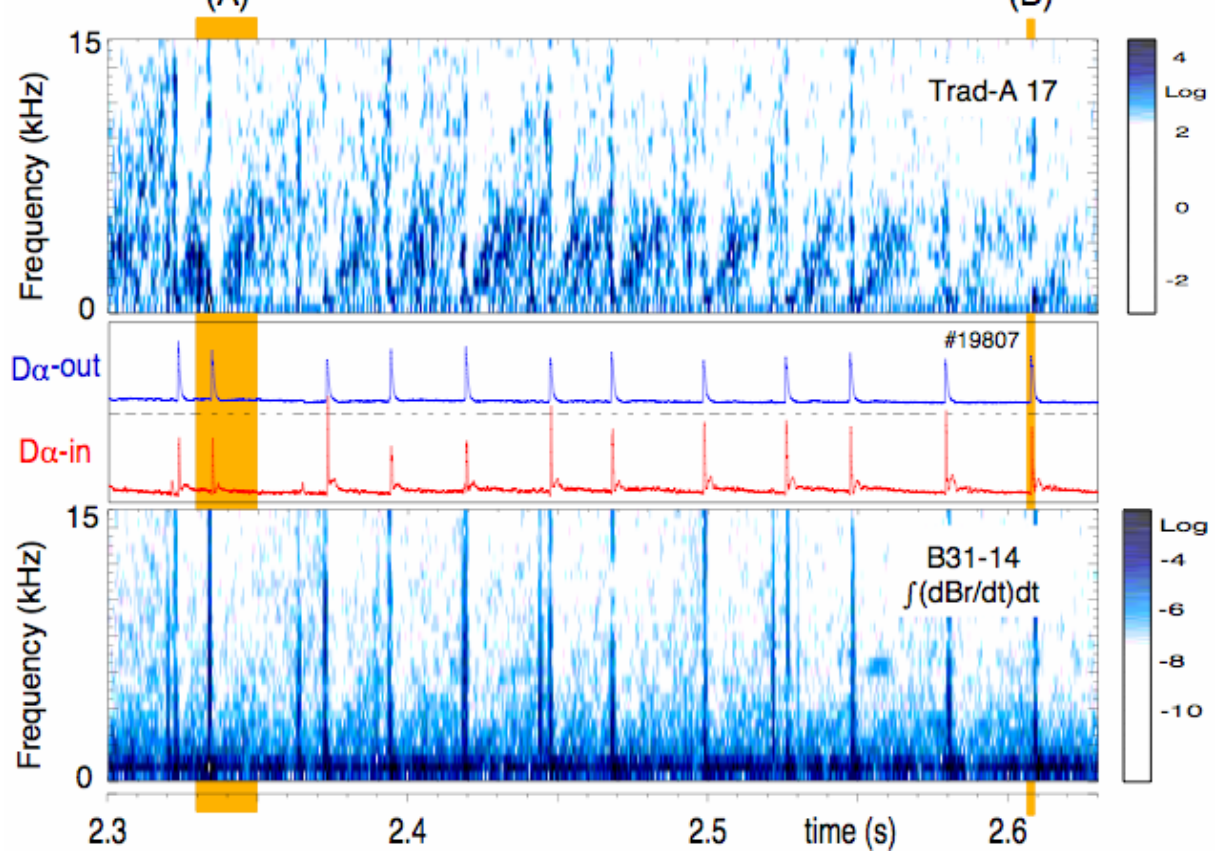

FIG.5. \# 19807: Low frequency spectrograms of the ECE radiation temperature in the edge barrier (top) and of the time integrated signal $B_{r}(t)$ of the magnetic pick-up coil B31-14 (bottom) in comparison with inner and outer divertor $D_{\alpha}$ emission (mid). The time slices (A) and (B) indicated by orange (or grey) vertical bars have been analysed already in context with figures 4 and 3, respectively,

Looking finally onto the remote divertor $\mathrm{D}_{\alpha}$ emission (figure 5 , mid), there is some low frequency noise specifically in the inner divertor with frequencies up to about $7 \mathrm{kHz}$. It is not clear yet whether this is due to upstream input modulated by the pedestal modes, or due to fluctuations driven independently by divertor physics effects.

\section{ELM induced filaments in the scrape-off layer}

For a limited number of shots, the mid plane manipulator, equipped with a multi-pin Langmuir head and/or magnetic pick-up coils, has been used to investigate the ELM induced filament structure and dynamics in the scrape-off layer wing and limiter vicinity $[18,19]$. More recently, a specific filament probe FP has been implemented near the manipulator on approximately the same magnetic flux bundle [20,21]. It combines a BAL-type magnetic 
probe (B31-30) with four Langmuir pins at the front and can be shifted out of the limiter shadow to investigate plasma filaments just in front of the limiter. In addition, a $2 \mathrm{~d}$ thermography camera viewing these probes and adjacent limiters is used to derive the heat load onto these elements. The distance between probes and plasma has been substantially varied by radial plasma position scans. Nearly identical radial decay lengths of about $2 \mathrm{~cm}$ have been found during ELMs for particle as well as for heat flux, indicating an elevated ion temperature well above electron temperature [20]. Correlating the four pins of the filament probe yielded a downward cross-field velocity of a few $\mathrm{km} / \mathrm{s}$ and vertical filament extension of several centimetres (equivalent to very high effective toroidal wave number approaching $\mathrm{n}_{\text {tor }} \sim 100$ ). The detailed probe signal variation indicates that these filaments are not smooth and uniform, but show a tendency to decay into even smaller substructures [20]. An attempt to model the magnetic signal in the nearby integrated pick-up coil by a dipole current in these 'open' filaments according to simple models [39] reproduced some qualitative features, but the required currents were by one to two orders of magnitude higher than the filament ion saturation current. Therefore the magnetic ELM signature is dominated by 'attached' pedestal structures, effectively masking the small SOL filament signals. Filaments are detectable on magnetic signals only in less turbulent phases.

Still the question remains, how these high-n filamentary structures observed in the SOL during ELMs are related to the low/medium-n electromagnetic pedestal perturbations described above. The following example may provide a preliminary answer. Figure 6 compares signals from the reciprocating probe (RP) and the filament probe (FP) in sectors 89 with those from the familiar poloidal magnetic pick-up coil array in sector 15. The same ELM (\#21292, $t=2.209 \mathrm{~s}$; parameters listed in table 1) has been chosen as in [20] (see figure 2 therein). Since the FP and RP are transiently positioned just in front of a limiter between them on approximately the same field line (figure 6, top), similar ion saturation currents are expected and measured by both probes, except for the late decay phase, as shown in the middle part of figure 6 . The FP magnetic signal $\left(\mathrm{dB}_{\mathrm{r}} / \mathrm{dt}\right.$; probe B31-30) shows the familiar ELM signatures described in previous sections. The overlaid vertical lines mark a few instants with more or less pronounced $\mathrm{dBr} / \mathrm{dt}$ maxima. The $\mathrm{j}_{\text {sat }}$ signal from pin 3 , located on the protection frame of B31-30, shows a rapid onset at or just after most of these lines. The delay from the $\mathrm{dB} / \mathrm{dt}$ maximum to the first $\mathrm{j}_{\text {sat }}$ maximum is a few tens of microseconds. For a radial distance of about $6 \mathrm{~cm}$ between pin and separatrix, this would correspond to radial filament velocities of up to several $1000 \mathrm{~m} / \mathrm{s}$, consistent with values quoted in the literature [2]. The individual $\mathrm{j}_{\text {sat }}$ bursts show significant substructure, probably due to subsequent filament overlap and/or disintegration of large filaments into smaller ones. This is still under investigation.

The question, whether the filament source can be directly related to rotating helical pedestal structures described earlier is addressed in figure 6 , bottom. Time integrated pick-up coil signals, $\mathrm{B}_{\mathrm{r}}(\mathrm{t})$, are shown for the poloidal probe array B31-05 to B31-10 (red-orange curves) in comparison with the FP probe B31-30 (black curve). As in previous figures 2 and $3, \mathrm{~B}_{\mathrm{r}}$ is set to zero at the window start and the slow global magnetic field variation is essentially due to the usual ELM induced inward equilibrium shift, approaching its maximum value of $\sim 8 \mathrm{~mm}$ at the time window end. The inclined dashed lines drawn across the $\mathrm{B}_{\mathrm{r}}(\mathrm{t})$ signals indicate a few pronounced upward rotating helical structures $(\sim 4 \mathrm{~km} / \mathrm{s})$. Here it is important to note that the top array probe, B31-10, is located about at the same poloidal height as the FP probe B3130. In fact, both signals exhibit a rather similar modulation, but with the FP signal delayed by 

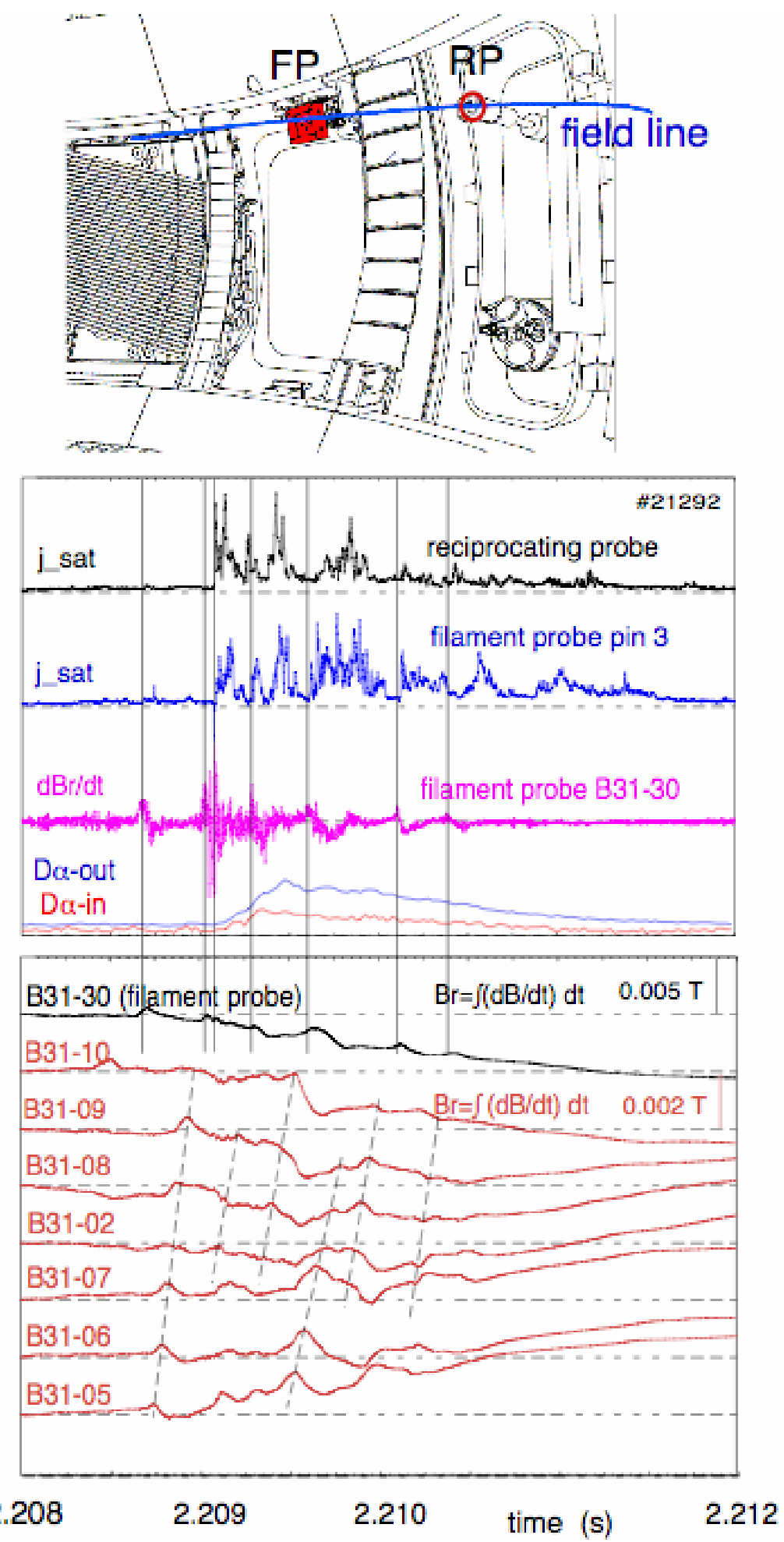

FIG. 6. Comparison of reciprocating probe (RP) and filament probe (FP) signals with magnetic pickup coil array signals. Top: RP and FP location on the LFS torus wall. Mid: Ion saturation currents $\left(j_{s a t}\right)$ from both probes, $d B / d t$ from $F P$, and inner/outer divertor $D_{\alpha}$ emission (arbitrary units). The vertical lines indicate a few instants of maximal $\mathrm{dB} / \mathrm{dt}$. Bottom: Time integrated pick-up coil signals, $B_{r}(t)$, from the poloidal probe array (B31-05 to 10; see figure 2) and from the same type of probe integrated in the FP (B31-30). The inclined dashed lines indicated upward rotating structures (3-4 $\mathrm{km} / \mathrm{s})$. 
about $150 \mu \mathrm{s}$. Since the poloidal array is about $5 \mathrm{~m}$ toroidally away from the FP in the cocurrent direction, this corresponds to a toroidal phase velocity of about $30 \mathrm{~km} / \mathrm{s}$ in countercurrent direction, consistent with the poloidal upward phase velocity seen on the array. This seems to hold also for occasionally happening inter-ELM events as indicated by the pre-ELM event in figure 6. Only late in the ELM decay phase, the mutual correlation between the two $\mathrm{j}_{\text {sat }}$ signals and magnetics is lost. In conclusion, the filament bursts seen on $\mathrm{j}_{\text {sat }}$ are clearly correlated with the low/medium-n modes rotating upward, i.e. in electron drift or pedestal ExB direction.

In contrast, whenever a poloidal cross field filament velocity can be derived during an ELM in the far SOL from the filament probe pins, it is downward with several $\mathrm{km} / \mathrm{s}$. The latter might in principle be consistent with the average toroidal mass velocity in the pedestal region of about $20 \mathrm{~km} / \mathrm{s}$ in co-current direction as measured by charge exchange recombination spectroscopy (CXRS): if a filament is split-off and isolated from a toroidally rotating plasma, it might continue to rotate toroidally with the initial mass velocity because of inertia. Remembering the helical filament shape, a downward poloidal velocity close to the one seen by the multi-pin filament probe is expected. A lower toroidal filament velocity has been derived from the toroidal filament footprint width on divertor target plates, but the inferred velocity depends strongly on the upstream filament model assumed, resulting in large systematic error bars.

\section{Discussion}

Several specific physics discussions and tentative interpretations have been already presented above together with the experimental results. In this section, we try to combine the most important experimental evidence described above and/or referenced in the initial survey into a common picture, to establish working hypothesises, and to compare with theoretical models as appropriate.

\subsection{Spontaneous type-I ELM cycle}

\subsubsection{Inter-ELM events, precursors and their role for ELM triggering}

A few ms after the previous ELM, the plasma edge usually reaches a nearly stationary, apparently quiescent and axisymmetric phase with a well-developed edge barrier and nearly constant pressure gradient. Looking into detail, however, there may be still some rise in density and temperature, and, in addition, we find a wide range of inter-ELM edge perturbations, ranging from finite amplitude fluctuations at a few $\mathrm{kHz}$ up to weakly structured broadband high-frequency noise approaching a $\mathrm{MHz}$ or so. We assume in the following that continuous core modes, if present, do not contribute to ELM release. Also modes located deep inside the barrier, but still in the ELM affected area and hence modulated by ELMs, are supposed to be of minor importance. Here, we are primarily interested in those inter-ELM perturbations residing essentially within the barrier. Two specific semi-coherent precursor modes have been investigated in more detail, the medium-n upward rotating washboard-type modes probably located around a rational barrier flux surface (section 3.2.1), and the strong low frequency plasma fluctuations in the steep gradient region slowly rotating downward or transiently locked to external asymmetries (section 4). However, neither of these localized precursor modes, sometimes even growing just before ELM onset, could be 
clearly identified as a necessary ELM trigger, since there are counter examples without them. In addition, singular events and spikes up to the size of mini-ELMs are often observed on magnetics, remote Langmuir probes and, if sufficiently large, even on divertor $\mathrm{D}_{\alpha}$, but still not triggering a large ELM. Whether the latter might be related to other ELM regimes (typeII, -III, grassy) remains to be analysed.

A major puzzle is still why a large type-I ELM eventually grows on a $10 \mu$ s time scale after many milliseconds with smoothly evolving average barrier profiles. Explosive instabilities have been invoked as a possible explanation [40]. In the presence of large scale fluctuations, however, there might be a simpler explanation. Assume that the driving force (e.g. the pressure gradient and/or the bootstrap current profile) slowly approaches the marginal limit, then the edge might be transiently (e.g. $<100 \mu \mathrm{s}$ ) driven by the macroscopic inter-ELM fluctuations sufficiently far beyond the marginal point so as to push a typical low-n instability to large amplitude within one precursor modulation period. The required fluctuation time scale and amplitude would be consistent with the several $\mathrm{kHz}, \sim 20 \%$ Thomson-ECE fluctuations, but seems to rule out the small amplitude washboard modes. Still the dominant energy reservoir driving the ELM remains unclear. According to the peeling-ballooning paradigm [41], the pressure gradient rise is supposed to be limited first by the onset of high-n ballooning turbulence. With increasing bootstrap current, essentially driven by the pressure gradient, but resistively delayed (resistive decay of the induced counter current), the ELM is then initiated via the rapid growth of a low-n external peeling mode, probably assisted by the macroscopic fluctuations. Our observations are qualitatively consistent with this picture, though the strong variability of the ELM cycle and inter-ELM activity, dependent on the actual discharge scenario, indicates that reality is still a bit more complex.

\subsubsection{Non-linear ELM evolution, edge profile erosion and recovery phase}

As we have seen, in the simplest case, an initial LFS helical filament structure may grow on a $10 \mu$ s scale out of some precursor noise with essentially random toroidal phase and low toroidal quasi-mode number, e.g. around four. More such structures are triggered then with a jitter of only a few tens of $\mu$ s [27]. This corresponds to the time needed by a shear Alfvén wave and/or by thermal edge electrons to travel several times poloidally (and factor q more toroidally) around a flux surface, communicating the initial edge perturbation essentially to the whole plasma surface. Like the higher-n washboard-type precursor modes, these low-n structures generally rotate upward at the LFS, i.e. in the electron drift or barrier ExB drift direction. The latter is assumed to be the dominant mechanism determining linear MHD mode rotation, and the measured velocities are consistent with typical radial electric fields in the steep gradient region. The helical mode amplitudes obtained are sufficiently large to influence the LFS ion cyclotron antenna coupling. In fact, a toroidal counter-current phase velocity of order $100 \mathrm{~km} / \mathrm{s}$ has been derived from the ELM onset response of the four toroidally distributed antennae, consistent with the poloidal upward velocity seen on the poloidal array, and also with the LFS ballooning mode amplitude estimated from the absolute radial magnetic field perturbation (order of perpendicular pressure decay length; section 3.2.2).

Up to and around the $\mathrm{dB} / \mathrm{dt}$ maximum (few $100 \mu \mathrm{s}$ ), these non-linear $3 \mathrm{~d}$ edge perturbations modify precursor modes and drive secondary modes at various frequencies and wavelengths. These cause, on one hand, enhanced turbulent cross field transport and divertor load in the hot scrape-off layer near the distorted and possibly ergodized plasma surface, and, on the 
other hand, macroscopic filament ejection and radial drift, forming on average a scrape-off layer wing with a few $\mathrm{cm}$ decay length [20]. In turn, both of these plasma loss mechanisms may contribute the primary mode saturation and damping. Therefore, with progressive edge profile erosion, $\mathrm{dB} / \mathrm{dt}$ decays, but $\mathrm{B}_{\mathrm{r}}(\mathrm{t})$ still indicates rotating $3 \mathrm{~d}$ plasma perturbations, correlated with bursts of ejected filaments. In due course, all non-axisymmetric, electromagnetic deformations seem to die away and the correlation with residual particle bursts received by SOL wing Langmuir probes is lost. About 1 to $2 \mathrm{~ms}$ after ELM onset (depending on shot scenario) a fairly quiescent, relaxed edge state is reached with moderately flattened temperature, density and pressure profiles [16,11]. Though not yet measured in detail, the edge current profile has most probably flattened also. After a few more milliseconds of profile recovery, the quasi-stationary fluctuating inter-ELM phase is reached again and the ELM cycle as described closes.

\subsubsection{Filament detachment and drift dynamics}

Ejected filaments have been studied on ASDEX Upgrade using a variety of diagnostic techniques. Rather detailed information on the filament rate, amplitude and mode number evolution during ELMs has been obtained from filament footprints on divertor target plates $[27,28]$. These have been complemented by Langmuir probe, magnetic pick-up and thermography measurements in the SOL wing and on limiters [20], and, most recently, with a specific filament probe as described in section 5. A clear correlation between low-n rotating ELM pedestal structures and intermittent filament bursts arriving in the far SOL has been observed (section 5). In essence, small size filaments seem to be released from transient, toroidally rotating, helical pedestal structures specifically in that LFS region, where toroidal and helical curvature add up. Such a rotating light house picture seems to fit quite a few details seen e.g. in figure 6: When a narrow helical perturbation maximum passes the probe location, the fastest filaments $(\sim$ few $1000 \mathrm{~m} / \mathrm{s})$ arrive first with small time delay relative to the magnetic pedestal mode signal, gradually followed by the slower ones (down to $\sim 100$ $\mathrm{m} / \mathrm{s}$ ). Since the rotating source is toroidally extended, a mixture of velocities is expected later on in the probe signal, but difficult to disentangle in practice. In this model, there is also no real contradiction because of opposite mode and filament rotation: a secondary filament rapidly growing and detaching within the primary mode transit time simply continues to rotate with the initial co-current edge mass rotation.

The radial drift of detached filaments in contact with material walls on both ends can be qualitatively explained by a simple model based on partial short circuit of the vertical charge separation current along field lines through material walls. If this short circuit would be perfect, such an isolated filament would be in static equilibrium ('line tying' at the target plate). However, because of finite sheath resistance at the plasma-wall interface (essentially the linear part of a double Langmuir probe characteristic), there is a poloidal voltage drop across the filament, resulting in a residual radial ExB drift velocity, which increases with filament length, but decreases quadratically with vertical filament height [39]. This means that smaller filaments attain higher velocities, and that initially large filaments, once perturbed on a shorter scale, may easily disintegrate into several smaller fragments [42]. The latter effect may also help to explain the experimentally observed trend for progressive radial mode disintegration, i.e. from primary low-n ELM structures in the pedestal, to secondary ejected filaments of intermediate size (and toroidal quasi-mode numbers), further breaking up sequentially into several $\mathrm{cm}$ blobs.

In parallel to the radial filament motion, particles, energy and momentum are continuously 
lost along magnetic field lines, essentially by classical transport, resulting in a radial filament decay length [20], which can be checked against experiment. For instance, the measured radial density and equal power decay length of about $2 \mathrm{~cm}$ in the far SOL would be consistent with a majority of filaments having a radial velocity of a few $100 \mathrm{~m} / \mathrm{s}$, corresponding filament height of a few $\mathrm{cm}$ [39], and warm ions $(\sim 30-60 \mathrm{eV})$, but cold electrons $(\sim 10 \mathrm{eV})$ [20]. High electron temperature in the far SOL can be expected only for the fastest filaments (few $1000 \mathrm{~m} / \mathrm{s}$ ), consistent with a minority of hot SOL wing filaments seen by Thomson scattering [38]. These hot filaments may also contribute to the enhanced tungsten sputtering observed at LFS limiters during ELMs in competition with ELM enhanced fast ion loss [30].

The momentum exchange between filament and divertor targets along field lines is more difficult to assess. In principle, the partial 'line tying' effect sketched above with respect to radial drift motion (partial short circuit of poloidal electric field at the target plates) also applies for toroidal and/or poloidal rotation, i.e. partial short circuit of the radial electric field driven by the poloidal cross field filament velocity component. A first estimate indicates a filament slowing down time of the order of the radial filament drift time. Therefore, a more quantitative assessment is clearly desirable.

Despite the obvious correlation between pedestal modes and filament ejection, the detailed mechanisms behind the filament's detachment from the separatrix-limited hot and rotating edge plasma remains open. Starting from static profiles with super-critical edge gradients, various theoretical models and numerical codes show multiple finger-like, eventually detaching structures with some similarity to our problem [40,43-45]. There is also a huge amount of literature on edge turbulence, blob transport etc, which might be related to certain filament aspects discussed above. But even a superficial comparison with our data is beyond the scope of this type-I ELM paper.

\subsection{Pellet induced versus spontaneous ELMs}

Dedicated ELM studies with the HFS pellet injection tool, mostly done in the perturbative mode of operation $\left(f_{\text {pellet }}<<f_{\text {ELM }}\right.$ ), have shown that an ELM is triggered as soon as the ablating pellet arrives at the pedestal top, or even slightly before [11,33,34,37]. Pellet ablation and particle deposition beyond this radius is virtually irrelevant for ELM triggering, but contributes to core fuelling and transport delayed axisymmetric edge modification. Accordingly, the difference between pellet induced and spontaneous ELMs depends on pellet size and velocity, which both have been varied over a wide range by centrifuge input settings plus statistically varying mass loss in the guiding tube to HFS. In the case of large pellets as applied in previous fuelling studies, the promptly triggered ELM tends to be longer with larger integral particle loss. In addition, this prompt ELM is quickly followed by a cascade of several satellite ELMs, expelling part of the excess edge particle inventory in a repetitive manner. In contrast, for the smallest pellets (or pellet fragments) available so far on ASDEX Upgrade, the basic ELM signature approaches that of spontaneous ones. Apart from the macroscopic pedestal mode structure, this applies even for edge fluctuation spectra measured by reflectometry and the target plate footprints derived from thermography [9]. Since still each pellet safely triggers an ELM, even smaller pellets might be sufficient for ELM pacing. The specific pellet case shown in fig. 2 is somewhere in between the extreme cases, with one satellite ELM after the prompt one.

Still, the detailed pellet-ELM trigger mechanism is not yet clear. Apart from the short recovery phase, ELMs can be triggered at any time during a spontaneous ELM cycle with 
constant time delay relative to pellet arrival at the pedestal. This delay time is $\sim 50 \square \mu \mathrm{s}$, probably too short for the cold pellet ablation plasmoid to expand along field lines from the HFS injection point to the LFS, where ELMs usually start [34,37]. Furthermore, the toroidal phase of the rotating LFS structures seen by the LFS pick-up coil array after ELM onset varies statistically over a number of pellet ELMs despite fixed injection geometry (section 3.2.3). Together, these observations indicate that it is not the ablation plasmoid itself which simply grows into an ELM.

Discussing possible trigger mechanisms, one should remember that pellets induce strong local perturbations of pressure, magnetic field, electron heat flux, toroidal momentum, resistivity and electric potential, all of them possibly players in the ELM release game. For large fueling pellets with $1.7-4.810^{20} \mathrm{D}$ atoms penetrating far beyond the barrier, peak plasmoid parameters of $\left\{\mathrm{Te} \sim\right.$ few eV, $\mathrm{n} \sim$ few $\left.10^{23} \mathrm{~m}^{-3}, \beta \sim 10 \%\right\}$ have been reported [46]. Less spectacular local plasmoid values are expected, though not yet directly measured, in the pedestal during pellet passage, especially for the smaller $510^{19} \mathrm{D}$ atom pellet case shown in figure 2 . The pellet induced perturbations are quickly communicated around the torus along field lines on a shear Alfvén and/or electron transit time scale, both of order $10 \mu$ s for one poloidal (and q toroidal) revolutions. It is not possible yet to sort out the dominant trigger effect. One should notice, however, that with such extreme local parameters new variants of peeling-ballooning modes may be expected. For instance, the high ohmic resistance of the toroidally expanding pellet ablation cigar should greatly facilitate field line reconnection and speed up resistive mode growth and island formation. Altogether, the ability to promptly trigger an ELM at any time in the ELM cycle may just be due to the excessive pellet perturbation with presently available pellet sizes (relative to the intrinsic inter-ELM fluctuations described above). Therefore, following the peeling-ballooning paradigm, a trigger threshold gradually increasing with rising edge current in between ELMs may be expected for pellet sizes near the ultimate lower mass limit.

In contrast to perturbative mode, in ELM pacing mode each ELM is initiated by a pellet ( $f_{\text {pellet }}$ $=\mathrm{f}_{\mathrm{ELM}}$ ) and the average background plasma profiles from edge to core adjust self-consistently to the repetitive pellet source on a global particle confinement time scale. In view of future ELM pacing and mitigation applications, however, it is more interesting to compare spontaneous and induced ELMs for essentially identical edge pedestal conditions by appropriately enhanced gas puffing in the non-pellet case [47]. In both cases, the qualitative ELM features are recovered, but the mitigation effect and its origin is easier to isolate in the latter.

\section{Summary and conclusions}

A large database on structure and dynamics of spontaneous as well as pellet induced ELMs has been accumulated on ASDEX Upgrade during past experimental campaigns. An overview of the available material has been given and most recent advances in experiment and data analysis have been described in some detail. An attempt has been made to construct a common spatio-temporal picture of the ELM cycle, without and with pellets, from this combined database, with the routinely available fast magnetic pick-up coil arrays providing a key link between various observations.

A large variety of inter-ELM and precursor activity has been observed in a wide mode 
number and frequency range, e.g. semi-coherent washboard-type modes (around $n \sim 8$ ) below $100 \mathrm{kHz}$, rotating in the electron drift or, physically more relevant, in the pedestal ExB direction, and nearly locked, large amplitude $(\sim 20 \%)$ intermediate-n pedestal fluctuations in the several $\mathrm{kHz}$ range. Though no 'necessary' ELM trigger could be identified, these modes, especially the strong low frequency fluctuations, might be non-linearly involved in sudden spontaneous ELM onset.

The initial dynamic ELM phase generally shows more or less incoherent low-n (near $n \sim 4$ ) helical mode structures, growing on a $10 \mu$ s scale, rotating again in the pedestal ExB direction and saturating at an amplitude comparable to typical edge gradient lengths. With progressive $3 \mathrm{~d}$ edge profile erosion this primary mode activity becomes more complex and irregular, depending on actual shot scenario and pedestal parameters.

In parallel to the rapid onset and saturation of the primary electromagnetic pedestal mode activity, transport into the SOL and divertor rises rapidly, and bursts of filaments are ejected into the far scrape-off layer, with the average particle flux arriving at limiters increasing an order of magnitude above inter-ELM blobby transport. A rather clear correlation of secondary filament bursts with the primary rotating pedestal modes has been found. The burst substructure and far SOL filament sizes indicate successive filament fragmentation. With radial velocities up to few $1000 \mathrm{~m} / \mathrm{s}$, at least ions remain hot and may contribute to (tungsten coated) limiter erosion. Non-axisymmetric filament footprints at divertor target plates, representing filaments shortly after detachment from the hot pedestal, reflect the initial low-n pedestal mode structure, but show increasing toroidal quasi-mode number later on, approaching values compatible with LFS limiter footprints.

After partial edge profile relaxation, a quiescent recovery phase of a few ms follows with nearly vanishing electromagnetic activity all over the spectral range. This occurs despite the fact that the radial pressure gradient in the barrier, while reduced at this instant, is still significant (ref. [16], figure 3). With gradually restoring H-mode barrier gradients the ELM cycle closes.

Pellet induced ELMs behave qualitatively similarly to spontaneous ones, at least for the smallest pellets available so far. Though the pellet clearly acts as the principal ELM trigger, the dominant physical mechanisms behind remain still open, since the ELM starts at random toroidal phase relative to the pellet launch position. In view of the extreme local edge perturbation induced by the pellets so far, it is not so surprising that an ELM can be initiated practically at any time within the ELM cycle. We anticipate that with further decreasing pellet size towards a lower critical mass limit, the probability of triggering an ELM will again increase with the time elapsed after the previous one.

Though a comprehensive theoretical ELM description in realistic divertor tokamak geometry is not yet available, there are various ideas and simplified models available in the literature, which are useful for qualitative comparison with our - also incomplete - experimental evidence. Globally, the ELM cycle sketched above may be still consistent with the standard peeling-ballooning paradigm [41], if we assume that the edge bootstrap current profile, not yet determined experimentally, becomes effective only on a resistive pedestal time scale, i.e. much slower than the pressure gradient recovery. Some evidence to support this idea has been recently reported, based on current profile measurements with a lithium beam [48]. In this model, the ELM is associated with a current driven low-n external kink or some more realistic variant of it. In this picture, the inter-ELM washboard-type precursor modes might 
be seen as some sort of internal peeling-ballooning mode saturating at finite amplitude.

The detached filament drift in the far SOL is reasonably understood in terms of simple electrostatic models involving partial short circuit of the curvature driven charge separation through the electrostatic sheath at wall intersection [39]. The filament detachment from the hot pedestal plasma across the separatrix, however, is much more complicated. Because of strong x-point shear, the filament cross section becomes extremely elongated there and beyond (remember the thin toroidally stretched spiral footprints on the divertor target [27]), with the small dimension inevitably reaching ion gyro radius dimensions. Therefore, a rigorous description of this process must await non-linear and non-local multi-scale edge turbulence codes in realistic separatrix geometry as discussed e.g. in [45].

As mentioned in the introduction, this paper has been focused on the combined analysis of ELM related results obtained from a large number of different diagnostics taken exclusively from ASDEX Upgrade. In view of the wide variety of discharge scenarios run in such a machine and the rather small fraction of dedicated discharges with optimal, problem-oriented diagnostic settings, this is already a challenge by itself. The approach mainly chosen here was to use the routinely available fast magnetic probe system, specifically the poloidal LFS 'ballooning' probe array as a common reference (installed years ago specifically for such time and space resolved electromagnetic edge measurements near the 'bad curvature' plasma surface). This strategy cannot be trivially extended to inter-machine comparison, since the desired information is usually not available in sufficient detail or requires a much more direct interaction between experimental teams. The latter has been undertaken in an ASDEX Upgrade - MAST comparison based primarily on Langmuir and optical diagnostics [18]. A more detailed comparison with other large tokamak ELM results is beyond the scope of this paper. Instead, the reader may be again referred to a recent survey of type-I ELM dynamics measurements [2] in a number of large tokamaks including ASDEX Upgrade.

\section{References}

[1] Federici G. et al 2003 Plasma Phys. Control. Fusion 451523

[2] Leonard A.W. et al 2006 Plasma Phys. Control. Fusion 48 A149

[3] Neuhauser J. et al 2006 Structure and Dynamics of Spontaneous and Induced ELMs on ASDEX Upgrade, in Fusion Energy 2006 (Proc 21st Int. Conf. Chengdu, 2006) (Vienna: IAEA) CD-ROM file EX/P8-2 and http://www-naweb.iaea.org/napc/physics/FEC/FEC2006/html/index.htm

[4] Zohm H. 1996 Plasma Phys. Control. Fusion 38105

[5] Zohm H. et al 1996 Study of H-mode physics in ASDEX Upgrade, in Fusion Energy 1996 (Proc. $16^{\text {th }}$ Int. Conf. Montreal, 1996), Vol.1, p.439, IAEA, Vienna (1997)

[6] Suttrop W. et al 2003 Fusion Science \& Techn. 44636

[7] Suttrop W. et al 2000 Plasma Phys. Control. Fusion 42 A1

[8] Horton L.D. et al 2005 Nucl. Fusion 45856

[9] Lang P.T. et al 2004 Nucl. Fusion 44665

[10] Lang P.T. et al 2005 Nucl. Fusion 45502

[11] Nunes I. et al 2004 Nucl. Fusion 44883

[12] Fattorini L. et al 2005 High spatial and temporal resolution FM-CW reflectometry to study pellet triggered ELMs at ASDEX Upgrade Proc. $32^{\text {th }}$ EPS Conf. Plasma Physics (Taragona, Spain) vol 29C (ECA) P-5.089

[13] Conway G.D. et al 2004 Plasma Phys. Control. Fusion 46951 
[14] Conway G.D. et al 2006 Study of turbulence and radial electric field transitions in ASDEX Upgrade using Doppler reflectometry, in Fusion Energy 2006 (Proc. 21st Int. Conf. Chengdu, 2006) (Vienna: IAEA) CD-ROM file EX/2-1 and http://www-naweb.iaea.org/napc/physics/FEC/FEC2006/html/index.htm

[15] Kurzan B. et al 2005 Phys. Rev. Lett. 95145001

[16] Kurzan B. et al 2007 Plasma Phys. Control. Fusion 49825

[17] Reich M. et al 2004 Plasma Phys. Control. Fusion 46797

[18] Kirk A. et al 2005 Plasma Phys. Control. Fusion 47995

[19] Herrmann A. et al 2004 Plasma Phys. Control. Fusion 46971

[20] Herrmann A. et al 2007 J. Nucl. Mater. 363-365 528

[21] Schmid A. et al 2007 Rev. Sci. Intrum. 78053502

[22] Tsalas M. et al 2007 J. Nucl. Mater. 363-365 1093

[23] Tsalas M. et al 2005 J. Nucl. Mater. 337-339 751

[24] Koch B. et al 2007 J. Nucl. Mater. 363-365 1056

[25] Mueller H.W. et al 2002 Profile and transport studies in the outer scrape-off layer at ASDEX Upgrade Proc. $29^{\text {th }}$ EPS Conf. Plasma Physics (Montreux, Switzerland) vol 26B (ECA) O-2.06

[26] Bobkov V. et al 2003 Interaction of ICRH electric fields and the sol plasma in ASDEX Upgrade Proc. $30^{\text {th }}$ EPS Conf. Plasma Physics (Petersburg, Russia)

vol 27A (ECA) P-1.165

[27] Eich T. et al 2003 Phys. Rev. Lett. 91195003

[28] Eich T. et al 2005 Plasma Phys. Control. Fusion 47815

[29] Herrmann A. et al 2003 J. Nucl. Mater. 313-316 759

[30] Dux R. et al 2007 J. Nucl. Mater. 363-365 112

[31] Garcia-Munoz M. 2006 Fast response scintillator based detector for MHD induced energetic ion losses in ASDEX Upgrade PhD Thesis, Ludwig-MaximiliansUniversitaet, Munich, Germany

[32] Kocsis G. et al 2004 Rev. Sci. Intrum. 754754

[33] Lang P.T. et al 2005 Czech. J. Phys. 551557

[34] Lang P.T. et al 2006 Plasma Phys. Control. Fusion 48 A141

[35] Smeulders P. et al 1999 Plasma Phys. Control. Fusion 411303

[36] Perez C.P. et al 2004 Plasma Phys. Control. Fusion 4661

[37] Kocsis G. et al 2007 Nucl. Fusion 471166

[38] Neuhauser J. et al 2002 Plasma Phys. Control. Fusion 44855

[39] Krasheninnikov S.I. 2001 Phys. Lett. A 283368

[40] Cowley S.C. et al 2003 Plasma Phys. Control. Fusion 45 A31

[41] Wilson H.R. et al 1999 Phys. Plasmas 61925

[42] D'Ippolito D. A. et al 2004 Contrib. Plasma Phys. 44205

[43] Wilson H.R., Cowley, S.C. 2004 Phys. Rev. Lett. 92 (2004) 175006

[44] Snyder P. et al 2005 Phys. Plasmas 12056115

[45] Scott B. et al 2006 Studies of the tokamak edge with self consistent turbulence, equilibrium, and flows, in Fusion Energy 2006 (Proc. 21st Int. Conf. Chengdu, 2006) (Vienna: IAEA) CD-ROM file TH/1-1 and http://www-naweb.iaea.org/napc/physics/FEC/FEC2006/html/index.htm

[46] Mueller H. W. et al 2002 Nucl. Fusion 42301

[47] Urano H. et al 2004 Plasma Phys. Control. Fusion 46 A315

[48] Thomas, D.M. et al 2006 Edge Current Growth and Saturation during the Type I ELM Cycle Proc. $33^{\text {th }}$ EPS Conf. Plasma Physics (Rome, Italy) vol 30I (ECA) P-5.139 